\title{
Automatic Alignment of Brain MR Scout Scans Using Data-adaptive Multi-structural Model
}

\author{
Ting Chen ${ }^{1, \star}$, Yiqiang Zhan ${ }^{2}$, Shaoting Zhang ${ }^{3, \star}$, and Maneesh Dewan ${ }^{2}$ \\ 1 Department of CISE, University of Florida, Gainesville, FL USA \\ tichen@cise.ufl.edu \\ 2 SYNGO US R\&D, Siemens Healthcare, Malvern, PA, USA \\ \{yiqiang. zhan, maneesh. dewan\} @siemens.com \\ 3 Department of Computer Science, Rutgers University, Piscataway, NJ, USA \\ shaoting@cs.rutgers .edu
}

\begin{abstract}
Accurate slice positioning of diagnostic MR brain images is clinically important due to their inherent anisotropic resolution. Recently, a low-res fast 3D "scout" scan has become popular as a prerequisite localizer for the positioning of these diagnostic high-res images on relevant anatomies. Automation of this "scout" scan alignment needs to be highly robust, accurate and reproducible, which can not be achieved by existing methods such as voxel-based registration. Although recently proposed "Learning Ensembles of Anatomical Patterns (LEAP)" framework 4 paves the way to high robustness through redundant anatomy feature detections, the "somewhat conflicting" accuracy and reproducibility goals can not be satisfied simultaneously from the single model-based alignment perspective. Hence, we present a data adaptive multi-structural model based registration algorithm to achieve these joint goals. We validate our system on a large number of clinical data sets (731 adult and 100 pediatric brain MRI scans). Our algorithm demonstrates $>99.5 \%$ robustness with high accuracy. The reproducibility is $<0.32^{\circ}$ for rotation and $<0.27 \mathrm{~mm}$ for translation on average within multiple follow-up scans for the same patient.
\end{abstract}

\section{Introduction}

The inherent MR imaging characteristics of high "in-plane" and low "out-ofplane" resolution warrant high accuracy requirements on the positioning of diagnostic imaging slices. Moreover, there is significant intra- and inter-patient variation in the starting orientations and axes of the scanning volumes. Hence, the use of low-res isotropic 3D "scout" scans as a pre-requisite sequence to improve the positioning accuracy has become a necessity prior to all clinical brain studies. In the current MR brain workflow (Fig[1(a)), a well-trained technician positions the imaging planes for the following high-res scans based on anatomy information in this fast low-res "scout" scan. For example, the axial plane of a standard high-res brain scan should be positioned parallel to the bicommissural line linking the landmarks of anterior and posterior commissure.

\footnotetext{
* This research was conducted while the authors were at Siemens Healthcare, USA.
} 

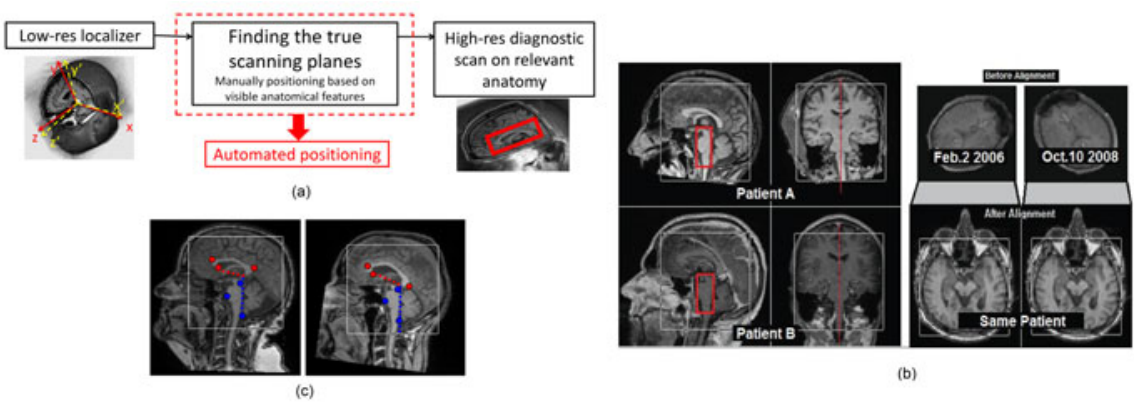

(b)

Fig. 1. (a) The 3 steps of the current MRI examination workflow. The goal of our is to develop an automatic algorithm for the $2^{\text {nd }}$ step. (b) Accuracy (left): a precise and standardized anatomy for all patients characterized by vertical brainstem and correct middle sagittal plane (MSP). Reproducibility (right): a consistent alignment for the follow-up scans of the same patient characterized by "no motion" in rigid structures when switching between aligned re-scan "scout" images. (c) Atlas and subject scans with different relations between brain stem and corpus callosum.

The inaccuracy, irreproducibility and time consumption limitations of manual alignment make automatic alignment algorithms greatly desirable. For these automatic alignment algorithms to be clinically acceptable, they need to be not only robust and accurate, but also reproducible, i.e., the alignment of the follow-up scans of the same patient should be identical with respect to relevant anatomies. Fig:1(b) illustrates the accuracy and reproducibility requirements for standardized brain alignment.

In [1, Andre et al. proposed an automatic slice positioning technique by registering the scout scan with a pre-aligned one. This method is not robust when the images contain large growing tumor, field of view change, severe noise, image artifacts or missing structures, etc. In addition, it is well known that the voxelbased image registration methods show high reproducibility in registering scans of the same patient, but are not as good for aligning scans from different patients, especially under the constraints of rigid or affine transformation for brain images. We experimentally validated this by registering several pair of re-scans ( $A_{1}$ and $A_{2}$ ) from the same patient to a model scan $(B)$ from a different patient using mutual information (MI) based image registration. Unfortunately, when both scans $\left(A_{1}\right.$ and $\left.A_{2}\right)$ achieve "best" matching with the model scan $(B)$, i.e., MI is maximized, the reproducibility is not good - larger motion was observed when switching between the two aligned scans $\left(A_{1}^{\prime}\right.$ and $\left.A_{2}^{\prime}\right)$.

Sharp et al. [2] proposed another approach jointly based on feature landmarks and image registration that relies on the availability of previous scans of the same patient. This method obtains remarkable accuracy and efficiency, however, their assumption on previous scan availability may not be applicable in real practice. In [3, Zhang et al. presented an auto-align system using image registration and active shape model, however, the "reproducibility" issue is not well addressed. 
Recently, "Learning Ensembles of Anatomical Patterns (LEAP)" 4] has shown high robustness in anatomy detection through learning redundant local appearance cues and sparse configurational models. Hence, an auto-align system based on LEAP [7] achieves extremely high robustness in the presence of severe artifacts/diseases. However, as 7] rigidly aligns scout scans to a single atlas/model, it fails to fully accomplish the "somewhat conflicting" "accuracy" and "reproducibility" goals simultaneously. To illustrate this, a schematic example is shown in Fig. 1(c). Here the brain stem and corpus callosum have different articulation angles (between blue and red dash lines) in the atlas and subject scans. If the alignment relies on all landmarks ( 3 from brain stem and 3 from corpus callosum), higher reproducibility is expected as small variations of landmark detections can be averaged out by robust point set registration techniques.

However, the accuracy requirement would be hard to meet (the aligned brain stem is not vertical) owing to the compromise of corpus callosum (note that only rigid alignment is allowed in MR auto-align). On the contrary, if the alignment relies only on the 3 landmarks of brain stem, one might achieve accurate alignment (vertical brain stem); but reproducibility is sacrificed as variations of brain stem landmarks have larger impact on the final alignment. Clearly, this conflict will not exist if the articulations between brain stem and corpus callosum are similar in atlas and subject scan or in other words, if the atlas constructed is adaptive to the detected anatomical feature landmarks in the subject scan. Indeed, this is the motivatation behind our Data-adaptive Multi-structural Model (DMM). It aims to compose a virtual atlas using learned exemplars of different local brain structures, based on the auto-detected anatomies in the subject scan. The constructed virtual atlas might not exist in real world, but it is the best approximation of the subject brain structure. Therefore it is a much better solution than picking from multiple models directly, given the limited data in practice. Additionally, multiple models would not be able to capture the local structure variations as well as the DMM. The philosophy behind our method is similar to [8]. However, our method differs significantly from [8] in terms of multistructural model (landmark based vs. image based) and objectives (alignment reproducibility vs. segmentation accuracy).

\section{Methodology}

Our auto-align system consists of three major steps. First, a set of anatomical landmarks are detected using LEAP 4 algorithm. Briefly put, LEAP is a learning-based algorithm that exploits "redundant" local appearance cues and sparse configuration models to achieve highly robust anatomy detections. Second, by querying the Data-adaptive Multi-structural Model (DMM) database, a virtual atlas is composed to optimally match the detected landmark set. Finally, a landmark-based rigid alignment is performed to register the landmark set with the virtual atlas, which brings the scout scan to the standard position. Since the major contribution of this work lies in the Data-adaptive Multi-structural Model (DMM), we will focus on it in the remainder of this section. 




Fig. 2. Flowchart for learning multi-structural clusters. We demonstrate the images in $2 \mathrm{D}$ while the data is actually in $3 \mathrm{D}$.

Notations. Define $\mathcal{I}=\{1,2, \ldots, M\}$ be the labels for $M$ anatomical landmarks. Given $N$ training scans with all these $M$ landmarks annotated, assume $x_{i}^{j}$ is the $i$ th landmark in the $j^{\text {th }}$ image, $\mathcal{X}^{j}=\left\{x_{i}^{j} \mid i=1,2, \ldots, M\right\}$ denotes a set including all landmarks from the $j^{\text {th }}$ image and $\mathcal{X}_{i}=\left\{x_{i}^{j} \mid j=1,2, \ldots, N\right\}$ denotes a set including the $i^{\text {th }}$ landmark from all $N$ images.

Construction of Multi-structural-Model. The basic idea of multi-structuralmodel is to group anatomical landmarks into different clusters. During the runtime, the "optimal" exemplars from different cluster can be composed to form a virtual model which has the similar brain structure spatial configuration as the subject's. To leverage the spatial correlations between landmarks, the construction of multi-structural-model starts from calculating the spatial variations.

Calculate spatial variations of each landmark: (Fig 2 (2)) To remove the variations from global transformation, we first register landmarks from different training images, i.e., $\mathcal{X}^{j}, j=1, \ldots N$, to a common canonical space. The spatially normalized point set is defined by $\widetilde{\mathcal{X}}^{j}=R_{j}\left(\mathcal{X}^{j}\right)$, where $R_{j}$ is a similarity transformation estimated using group-wise point set registration [5], which is a pre-step to align the training subjects. Given the average position of the $i^{\text {th }}$ landmark across all subjects $\overline{\mathcal{X}}_{i}=E\left(\widetilde{\mathcal{X}^{j}}\right)$, the spatial variation of the $i^{\text {th }}$ landmark can be captured by a displacement field $U_{i}=\left\{u_{i}^{j}=\widetilde{x}_{i}^{j}-\overline{\mathcal{X}}_{i} \mid j=1, \ldots, N\right\}$.

Build $3 D$ histogram of displacement fields: (Fig 2 (3)) To reveal the statistical insight of the spatial variations, a $3 D$ histogram $h_{i}$ is built on each displacement field $U_{i}$. More specifically, the whole space of displacement field is divided into $9 \times 9 \times 9$ bins along $x, y$ and $z$ dimensions and $h_{i}(x, y, z)$ is obtained by counting the occurrences of $u_{i}^{j}$ in bin $(x, y, z)$. As cumulative density function (cdf) is more robust than histogram, we convert $h_{i}(x, y, z)$ to cdf as $F_{i}(s, t, l)=\Sigma_{-\infty}^{s, t, l} h_{i}(x, y, z)$. It is used in the similarity measure in the next step. Landmark clustering using affinity propagation: (Fig 2 (4)) The clustering of anatomy primitives(landmarks) is finally accomplished by Affinity Propagation [6 method. Compared to other clustering methods, affinity propagation shows advantages in its less sensitivity to bad initialization and no requirement for a preset cluster number which is often unknown in our problem. Here, each landmark is considered as a data point in the affinity network and the similarity between label pairs $v$ and $w$ are defined as: 


$$
A(v, w)=-\alpha \sum_{s, t, l}\left\|F_{v}(s, t, l)-F_{w}(s, t, l)\right\|_{2}^{2}-(1-\alpha)\left\|\overline{\mathcal{X}}_{v}-\overline{\mathcal{X}}_{w}\right\|_{2}^{2}
$$

The second term measures the average distance between two landmarks. It ensures that two nearby landmarks have higher probability to be clustered together (in accordance with our observations of the brain anatomy).

As shown in Fig 3 , we now have a multi-structural database that consists of a set of clusters. In other words, brain is divided into multiple pseudo substructures (here "pseudo" indicates that a cluster might not

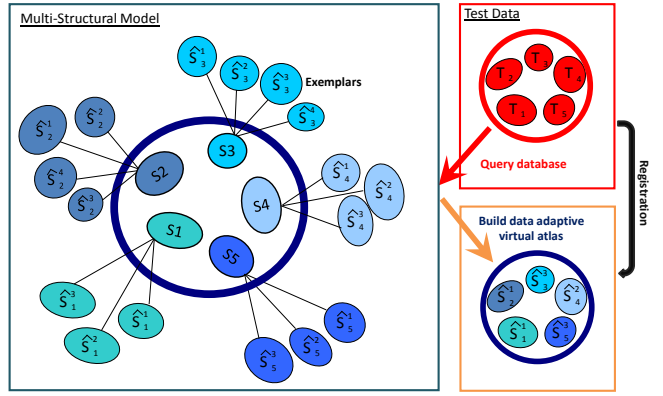

Fig. 3. This figure illustrates the Data-adaptive Multi-structural Model Database strictly correspond to the well defined sub-structures in the medical atlas). Each sub-structure has $N$ instances coming from the $N$ training images. More specifically, each $\mathcal{X}^{j}$ is now partitioned into a set of $\left\{\mathcal{S}_{k}^{j}\right\}, k \in\{1,2, \ldots K\}$, each of which contains a subset of landmarks from $\mathcal{X}^{j}$. These pseudo-sub-structures provide the flexibility of composing a virtual atlas while still maintaining the connections of closely related anatomy primitives.

Extraction of representative exemplars: Given the landmarks detected in a testing scout image, a naive way to compose an "optimal" virtual atlas from the multi-structural-model is to go through all instances in all clusters. Clearly, when the number of training samples become large, the runtime efficiency will be greatly degraded. Therefore, we propose to extract representative exemplars for each cluster $\mathcal{S}_{k}$. Principal Component Analysis is applied to all instances belonging to $\mathcal{S}_{k}$, i.e., $\left\{\mathcal{S}_{k}^{1} \ldots \mathcal{S}_{k}^{N}\right\}$. The first few principal variation modes $\widehat{\mathcal{S}}_{k}^{j}, j \in\{1,2, \ldots L\} ; L \ll N$, are used as representative exemplars of $\mathcal{S}_{k}$.

Adaptive Composition of Virtual Atlas. During run-time, multi-structural model is employed to derive optimal virtual atlas. Given a testing scout scan, our auto-align system starts by detecting a set of landmarks $T=\left\{t_{i}\right\}, i=1 \ldots N$ using LEAP. As LEAP algorithm has specific detectors for every anatomical landmark, the correspondences between detected landmarks and our multi-structural model are automatically built. $T$ is then decomposed into pseudo-sub-structures as $\left\{T_{k}\right\}, k=1 \ldots K$ according to the built-in landmark label clusters in the multi-structural model. For each $T_{k}$, it queries the multi-structural model and finds the most similar exemplars as $\widehat{S}_{k}^{\text {opt }} \leftarrow \min _{j}\left\|\mathcal{R}\left(T_{k}\right)-\widehat{S}_{k}^{j}\right\|_{2}$. Here $\mathcal{R}(\cdot)$ defines the transformation that brings $T_{p}$ to the multi-structural model space.

Finally, a virtual atlas $\mathcal{V}$ is obtained by integrating all these optimal substructural exemplars as $\mathcal{V}=\bigcup_{k=1 \ldots K} \widehat{S}_{k}^{o p t}$. As a result, each sub-structure of this atlas has the most similar spatial configurations with the detected anatomy 

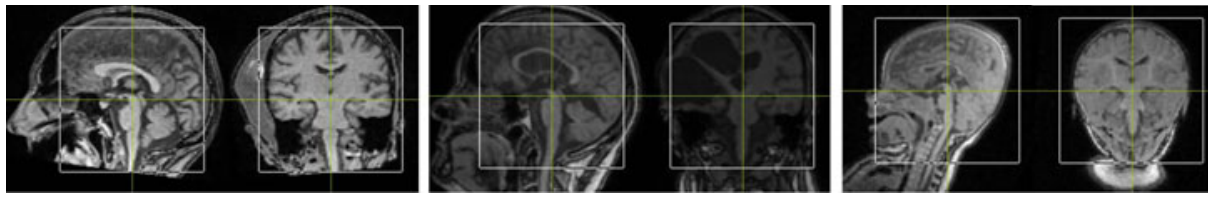

Fig. 4. Auto-align results on challenging cases, i.e., severe artifacts (Left), tumors (Center) and pediatric (Right). Please refer to Fig. 1(b) for alignment requirements.

primitives in the same local regions. As discussed in Section 1, if spatial configurations between different anatomy primitives are similar in atlas and subject, the accuracy and reproducibility requirements are no longer in conflict. Thus the virtual atlas derived from the multi-structural model paves the way to achieve accurate and reproducible alignment simultaneously.

\section{$3 \quad$ Experiments}

Experimental Setting. Our validation data set includes a large number of T1weighted $3 D$ scout scans: 731 adult (age: 15-87 years) and 100 pediatric (age: months to 14 years). Some adult volunteers are scanned multiple times to test the reproducibility of the algorithm. 90 adult and 40 pediatric scans were selected as training data. 22 anatomical landmarks are annotated for constructing the DMM model and training the LEAP algorithm. The regularization parameter $\alpha$ in Eqn. (11) was set to 0.7. We compare our method with the landmark-based alignment using Single Fixed Model (SFM), wherein the model is the average constructed by group-wise point set registration [5].

Accuracy. Our method provides accurate alignment on 690 cases out of the 701 testing cases. It was verified qualitatively by the experts who checked the correctness of the MSP and the verticality of the brainstem (as indicated in Fig. 1(b)). Some example alignment results on challenging cases in Fig 4 to demonstrate the robustness and accuracy of our algorithm. In Fig. 5, our method shows superiority accuracy with little $\left(<1^{\circ}\right)$ or no tilt, as compared to SFM, wherein brains brainstems are visibly slanted by $3^{\circ}-5^{\circ}$.

Reproducibility. We validate the reproducibility of our method on 5 volunteers, each with 14-18 re-scans. For better visualization, we overlayed two aligned re-scans in a checkerboard pattern in Fig 6. The edges of the brain structure (e.g.,
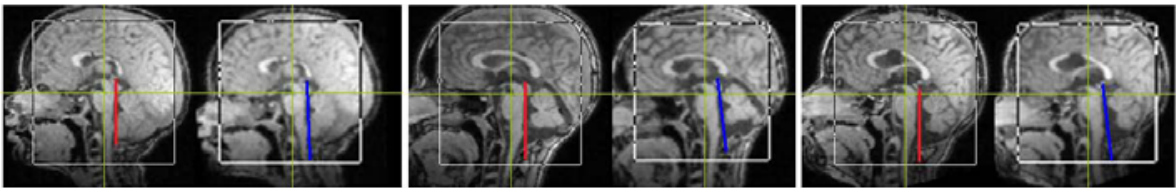

Fig. 5. Comparisons of $\mathrm{DMM}($ red) and SFM(blue) methods (in coronal views) 

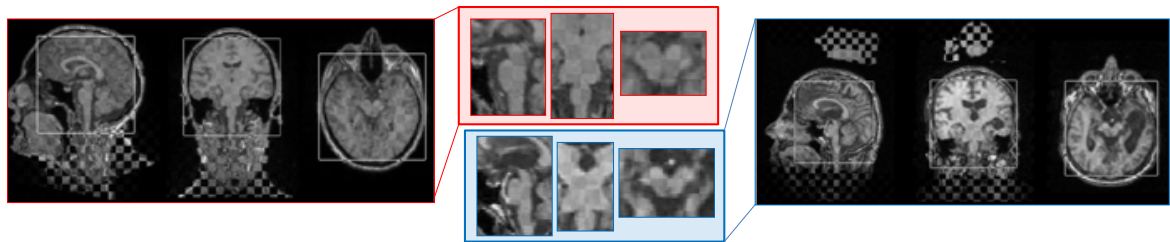

Fig. 6. Checkerboard visualization of aligned re-scans. Brainstem regions are zoomedin for a better visualization of reproducibility.
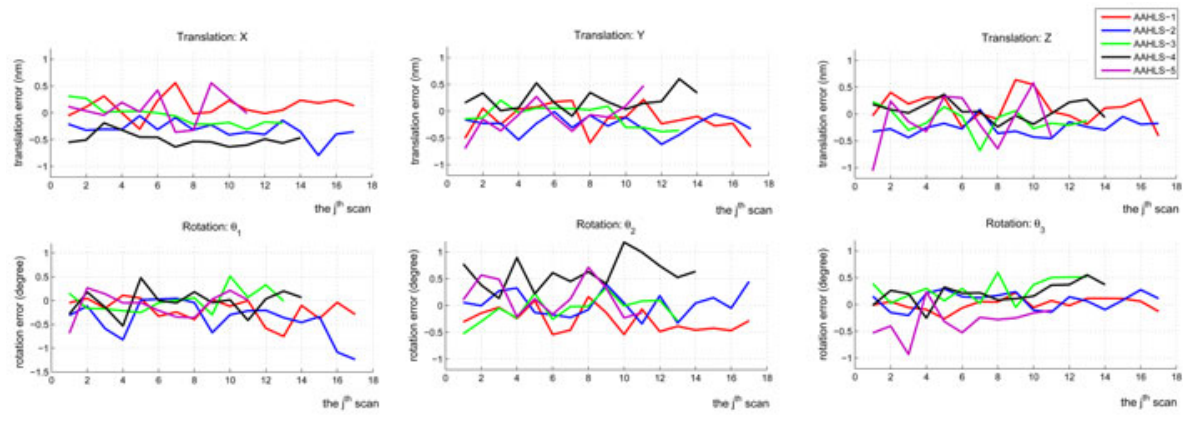

Fig. 7. Each figure shows the rotation or translation errors for each re-scan subject from the 5 data sets. The errors are estimated by registering each re-scan MRI localizer to a selected template from the same data set.

Table 1. Percentage of the re-scan data set with rotation error $\leq 0.1,0.2$ and 0.5 degrees, and the translation error $\leq 0.1,0.2$ and $0.5 \mathrm{~mm}$ (first three rows). The last three rows show the average rotation and translation errors along with the standard deviation and the improvements of DMM w.r.t SFM method.

\begin{tabular}{|c|c|c|c|c|c|c|c|c|c|c|c|c|}
\hline & \multicolumn{6}{|c|}{ Rotation Error (degree) } & \multicolumn{6}{|c|}{ Translation Error (mm) } \\
\hline & \multicolumn{3}{|c|}{$\overline{\mathrm{DMM}}$} & \multicolumn{3}{|c|}{ SFM } & \multicolumn{3}{|c|}{ DMM } & \multicolumn{3}{|c|}{ SFM } \\
\hline Statistics & $\theta_{1}$ & $\theta_{2}$ & $\theta_{3}$ & & $\theta_{2}$ & $\theta_{3}$ & $t_{x}$ & $t_{y}$ & $t_{z}$ & $t_{x}$ & $t_{y}$ & $t_{z}$ \\
\hline$\leq 0.1^{\circ} / \mathrm{mm}$ & $34.7 \%$ & $20.8 \%$ & $27.8 \%$ & $22.0 \%$ & $10.3 \%$ & $22.1 \%$ & $25.6 \%$ & $29.6 \%$ & $26.8 \%$ & $15.3 \%$ & $26.4 \%$ & $31.9 \%$ \\
\hline$\leq 0.2^{\circ} / \mathrm{mm}$ & $52.8 \%$ & $38.9 \%$ & $52.8 \%$ & $38.2 \%$ & $30.9 \%$ & $36.8 \%$ & $39.4 \%$ & $53.5 \%$ & $50.7 \%$ & $37.5 \%$ & $47.2 \%$ & $59.7 \%$ \\
\hline$\leq 0.5^{\circ} / \mathrm{mm}$ & $86.1 \%$ & $80.6 \%$ & $89.0 \%$ & $75.0 \%$ & $67.7 \%$ & $69.1 \%$ & $85.9 \%$ & $90.2 \%$ & $93.0 \%$ & $83.3 \%$ & $93.0 \%$ & $91.6 \%$ \\
\hline Abs. Mean & 0.238 & 0.318 & 0.231 & 0.313 & 0.580 & 0.379 & 0.268 & 0.226 & 0.244 & 0.309 & 0.253 & 0.277 \\
\hline Improvement & $23.96 \%$ & $45.17 \%$ & $39.05 \%$ & & & & $13.27 \%$ & $10.67 \%$ & $11.91 \%$ & & & \\
\hline STD & 0.093 & 0.174 & 0.108 & 0.074 & 0.260 & 0.107 & 0.143 & 0.037 & 0.092 & 0.157 & 0.098 & 0.134 \\
\hline
\end{tabular}

brain stems in the zoomed up images) crossing checkerboards are well preserved, which shows the good reproducibility of our method. To quantitatively evaluate the reproducibility of our algorithm, we obtain the "ground truth" transformation between re-scans using MI-based image registration, since it is well known that MI-based image registration is almost perfect in aligning scans from the same person. The quantitative "reproducibility" is shown in Fig 7. Table[1 shows the reproducibility of our method compared to SFM. For each re-scan dataset, 
we listed the percentage of scans at different error levels, the average translation and rotation errors, and the percentage of improvement from SFM to DMM.

Runtime Efficiency. Our method is implemented by Python2.5 and $\mathrm{C}++$. Running on an Intel(R) Xeon $(\mathrm{R})$ machine with $2.33 \mathrm{GHz} \mathrm{CPU}$ and $3 \mathrm{~GB}$ RAM, it takes about 5 seconds to align one $192 \times 192 \times 144$ brain MRI. This serves to anecdotally illustrate the computational time involved.

\section{Conclusions}

In this paper, we presented a method to automatically align MR brain "scout" scans with high accuracy, reproducibility and robustness. The joint goals of accuracy and reproducibility were met by using a data-adaptive multi-structural model. Our system, validated on a large number of clinical cases, automates the manually positioning procedure, which can highly improve the quality and speed of the brain MR examination workflow.

Acknowledgement. The authors would like to thank Martin Harder for a lot of valuable discussions and data collection.

\section{References}

1. van der Kouwe, A.J.W., Benner, T., Fischl, B., Schmitt, F., Salat, D.H., Harder, M., Sorensen, A.G., Dale, A.M.: On-line Automatic Slice Positioning for Brain MR Imaging. NeuroImage 27(1), 222-230 (2005)

2. Sharp, G.C., Kollipara, S., Madden, T., Jiang, S.B., Rosenthal, S.J.: Anatomic Feature-based Registration for Patient Set-up in Head and Neck Cancer Radiotherapy. Phys. Med. Biol. 50, 4667 (2005)

3. Zhang, L., Xu, Q., Chen, C., Novak, C.L.: Automated Alignment of MRI Brain Scan by Anatomic Landmarks. In: SPIE Med. Imag., vol. 7258 (2009)

4. Zhou, X.S., Peng, Z., Zhan, Y., Dewan, M., Jian, B., Krishnan, A., Tao, Y., Harder, M., Grosskopf, S., Feuerlein, U.: Redundancy, Redundancy, Redundancy: the Three Keys to Highly Robust Anatomical Parsing in Medical Images. In: Int. Conf. MIR, pp. 175-184 (2010)

5. Chen, T., Vemuri, B.C., Rangarajan, A., Eisenschenk, S.J.: Group-wise Point-set Registration using a Novel CDF-based Havrda-Charvat Divergence. Int. J. Comput. Vision 86(1), 111-124 (2010)

6. Frey, B.J., Dueck, D.: Clustering by Passing Messages Between Data Points. Science 315, 972-976 (2007)

7. Dewan, M., Zhan, Y., Peng, Z., Zhou, X.S.: Robust Algorithms for Anatomic Plane Primitive Detection in MR. In: SPIE Med. Imag., vol. 7260 (2009)

8. Shi, F., Yap, P.T., Fan, Y., Gilmore, J.H., Lin, W., Shen, D.: Construction of Multi-Region-Multi-Reference Atlases for Neonatal Brain MRI Segmentation. NeuroImage 51(2), 684-693 (2010) 